\title{
Relação das obras e revistas entradas durante $o$ ano de 1932, na Biblioteca da Faculdade.
}

POR COMPRA :

OBRAS

A. FERREIRA COELHO.- Codigo Civil dos Estados Unidos do Brasil. Vols. 1, 2, 3, 6, 7, 8, 11, 12, 13, 14, 15, 16, 17, $18,19,20,21,22$ e 23, Rio de Janeiro, 1920. 19 vols.

AFFONSO DE E. TAUNAY. - Historia Geral das Bandeiras. Paulistas. Tomos V e VI. 1929-1930. 2 Vols.

AGRIPINO VEADO. - Classificação decimal de Jurisprudencia (Sistema de fichas em cartolina) Rio de Janeiro, 1932 1 vol.

ALFREDO DE CARVALHO. - Bibliotéca Exotico-Brasileira. Rio de Janeiro, 1929-1930. 3 vols.

ANNUAIRE INTERPARLEMENTAIRE. - Paris. 1932. 1 vol.

ARAUJO VIANNA. - Novo Codigo Eleitoral do Brasil. S. Paulo, 1933. 1 vol.

BARTHE'LEMY (Joseph). - Traité élémentaire de Droit Constitutionnel. Paris 1926. 1 vol.

BEER (Max). - Histoire Générale da socialisme et des luttes sociales. Paris. 1932. 5 vols.

BIARDEAU (Laure). - Le Certificat Prénuptial. Paris, 1931. 
BLUM (René). - Les Assurances Aériennes. Paris, 19301 vol. BONNECASE (Julien). - Introduction á l'étude du Droit Paris, 1931. 1 vol.

CAILLOL (Georges). - Du Principe de l'inoposabilité des Exceptions au porteur d'un Effet de Commerce. Paris, 1931. 1 vol.

CALOGEROPANilos STRATIS (Spyros). - Le Pacte Général de Renonciation á la Guerre. Paris, 1931. 1 vol.

CASANOVA (Pierre). - L'Abandon de Famille en Droit Pénal Français. Paris 1930. 1 vol.

CAVALLERA (Ferdinand). - Précis de la Doctrine Sociale catholique. Paris, 1931. 1 vol.

CHABAS (Jean). - - De la déclaration de volonté en droit civil français. Paris. 1931. 1 vol.

CLASSIFICATION DE'CIMALE UNIVERSELLE. - Tomes 1 á III Bruxelles, 1927 á 1929. 1 vol.

CLOVIS BEVILAQUA. - Direito de Familia. Recife, 1908. 1 vol.

CLOVIS BEVILAQUA. - Direito das Successões. Rio de Janeiro, 1932.1 vol.

COLIN (Paul). - De la détermination du Mandat Salarié. Paris, 1931. 1 vol.

DECROLY E R. BUYSE. - Pratica dos Testes Mentais. Rio de Janeiro, 1931. 1 vol.

DECUGIS (Henri). - Traité Pratique des Sociétés par actions. Paris, 1931. 1 vol. 
DELPECH (Joseph) et JULIEN LAFERRIERE. - Les: Constitutions modernes. Paris, 1928-1932. 5 vols.

DRAGU (Thomas). - Juges-Citoyens ou Juges-de-Métier?, Paris, 1931. 1 vol.

DURAND (Paul). - Des Conventions d'irresponsabilité Paris, 1932. 1 vol.

ENCYCLOPEDIA OF THE SOCIAL SCIENCES. - New York, 1931. 8 volumes.

ENGELS (Fr.) - L'Origine de la Famille, de la Proprieté Privée et de l'Etat. Paris, 1931. 1 vol.

ESCHOLIER (Marc). - La Phonographie et le Droit d'au. teur, Paris. 1930. 1 vol.

ESPINOLA (Eduardo). - Anotações ao Codigo Civil Brasieiro. S. Paulo. 1929. 3 Vols.

EUGENIO DE CASTRO. - A Expedição de Martim Afonso de Souza. Rio de Janeiro, 1932. 1 vol.

FE'LIX (Maurice). - L'Activité économique de la Commune Paris, 1932. 1 vol.

FRANÇOIS (J.). - La Propriété de la Provision en matière de Lettre de Change. Paris. 1930. 1 vol.

GARDENAT (L.). - Traité de la Profession d'Avocat. Paris, 1931. 1 vol.

GIDE (Ch.). - Principes d'Economie Politique. Paris. 1931. 1 vol.

GONNARD (René). - Histoire des doctrines economiques Paris, 1930. 1 vol. 
GORDON (Ezequiel). - La responsabilité du chef de l'Etat dans la pratique constitutionelle récente. Paris, 1931.

GOYET (Francis). - Précis de Droit Pénal spécial. Paris, 1930. Broch. 1 vol.

GRANDJACQUES (Henri). - Les tendances internationales de l'Assurance Sociale. Paris, 1930. 1 vol.

GRZEGROZEWSKI (Ziemowit). - La lutte contre la mendicité et le vagabondage. Varsovia, 1930. 1 vol.

HANOTAUX (Gabriel). - Histoire de la France contemporaine (1871-1900). Paris. 4 vols.

HEITOR FURTADO DE MENDONÇA. - Primeira visitação do Santo Oficio ás partes do Brasil. Denunciações de Pernambuco. S. Paulo, 1929. 1 vol.

HO TCHONG CHAU. - Code Civil de la Répablique de Chine. Paris. 1930-1931. 2 vols.

INDEX GENERALIS. - Annuaire Général des Universités. Paris, 1932. 2 vols.

J. CAPISTRANO DE ABREU. - O Descobrimento do Brasil. 1929. 1 vol.

J. CAPISTRANO DE ABREU. - Capitulos da Historia Colonial. 1928. 1 vol.

J. CAPISTRANO DE ABREU. - Caminhos antigos e povoamento do Brasil. 1930. 1 vol.

JIMENEZ DE ASUA (LUIZ) e JOSE' AUTO'N ONECA. Derecho Penal. Madrid, 1929. 2 vols. 
JOSE' MARIA WHITAKER. - Letra de Cambio. 2a. edição revista e aumentada. S. Paulo, 1932. 1 vol.

JOSSERAND (Louis). - Cours de Droit Positif Français Paris, 1932. 3 vols.

KRAFT-EBING. - Psychopathie Sexuales, Paris, 1931. 1 vol.

LA GRESSAYE (Jean Brethe de). - Le syndicalisme. L'organisation professionelle et l'Etat. Paris. 1931. 1 vol.

LE BOUHEUR (A.) - La Responsabilité Civile en cas de Collision de Vehicules. Paris, 1931. 1 vol.

LECOMTE (André). - De la Nature Juridique des Ventes à option. Paris. 1931. 1 vol.

LEONEL FRANCA - O Divorcio. 2a. edição. Rio de Janeiro, 1931. 1 vol.

LE'VY (Edouard). - Traité pratique de législation des enfants naturels, simples, incestueux ou adultérins. Paris, 1926. 1 vol.

LOUIS (Paul). - Les Idées essentielles du Socialisme. Paris, 1931. 1 vol.

LOUP (Jean). - Les Sports et le Droit. Paris, 1930. 1 vol.

LUIZ DA CUNHA GONÇALVES. - Tratado de Direito Civil em comentario ao Codigo Civil Português. Coimbra, 1929-1930. 3 vols.

MACIEJEWSKI (Casimir). - La Théorie du Droit. Paris. 1931. 1 vol.

MAGNAN DE BORNIER (J.). - L'Empire Britannique. Paris, 1930. 1 vol. 
MAROTTE (Paul). - De l'application des droits d'auteur et d'artistes aux oeuvres cinématographiques et cinéphoniques. Paris, 1930. 1 vol.

MASPETIOL (Roland) et Pierre. - Le Tutelle Administrative. - Paris, 1930. 1 vol.

ME'ZE'ANDE (Henri) et LE'ON MEZE'ANDE. - Traité Théorique et Pratique de la Responsabilité Civile. Paris, 1931. 2 vols.

MIRKINE-GUETZEWITCH (B.). - Les Nouvelles Tendences du Droit Constitutionnel. Paris, 1931. 1 vol.

MIRKINE-GUETZEVITCH (B.). - Les déclarations des Droits de L'Homme. Paris, 1929. 1 vol.

MORI (Arturo). - Cronica de las Cortes Constituientes de la segunda Republica Española. Madrid, 1932. 6 vols.

MOUSKHELI (M.). - La Théorie Juridique de l'Etat Fédéral. Paris, 1931. 1 vol.

NIBOYET (J. P.) et P. GOULE. - Recueil de Textes Usuels de Droit International. Paris, 1930. 1 vol.

OCTAVIO KELLY - Codigo Eleitoral. Rio de Janeiro, 1932. 1 vol.

PATOUILLET (Jules). - Les Codes de la Russie Soviétique. Paris, 1929. 3 vols.

PERRIER (Paul). - Histoire de la Civilisation et de L'Esprit Humain. Paris, 1931, 1. vol.

PONTES DE MIRANDA. - Os fundamentos atuais do Direito Constitucional. Rio de Janeiro, 1932. 1 vol. 
POTULICKI (Michel). - Actes de la Conférence d'unification du Droit Pénal. Paris, 1929. 1 vol.

RABINOWICH (Léon). - Le Crime Passionnel. Paris, 1931. 1 vol.

REDSLOB (Robert). - Le Principe des Nationalités. Paris, 1930. 1 vol.

ROUAST (André). - La condition juridique de l'enfant conçu avant et né pendant le mariage. Paris, 1927. 1 vol.

RUI BARBOSA. - Comentarios á Constituição Federal Brasileira. S. Paulo, 1932. 1 vol.

SCELLE (G.) - Précis Elémentaire de Législation Industrielle. Paris, 1927. 1 vol.

STUDI DI DIRITTO ROMANO. - di Diritto Moderno e di Storia del Diritto. Pubblicati in onore di Vittorio Scialoja nel XXV anniversario di suo insegnamento. Milano, 1905. 2 vols.

TCHERNOFF (J.). - Traité de Droit Pénal Financier. Paris, 1931. 3 vols.

TCHERNOFF (J.). - Expertises judiciaires en matière pénale. Paris, 1932. 1 vol.

TISSIER (Pierre). - Traité des Assurances Sociales. Paris, 1931 1 vol.

TITO FULGENCIO. - Anotações ao Codigo Eleitoral. São Paulo, 1932. 1 vol.

TOBIAS MON'TEIRO. - Historia do Imperio. A Elaboração da Independencia. Rio de Janeiro, 1927. 1 vol. 
TREVELY (Joseph). - Essai sur l'Histoire et la situation du Sous-Marin en Droit International. Paris, 1931. 1 vol.

TURGEON (Charles). - Critique de la conception matérialiste de l'Histoire. Paris, 1931. 1 vol.

VALEUR (Robert). - La Responsabilité Pénale des Personnes Morales dans les Droits Français et Anglo-Américains. Paris. 1931. 1 vol.

VILLARD (Henri). - Le contrôle de L'Expertise Judiciaire en Matière Pénale. Paris. 1931. 1 vol.

VILLENEUVE (Marcel de la Bigue de). - Traité Général de l'Etat. Paris, 1929 e 1930 2a. edição. 2 vols.

VRABIESCO (Jeane-Eugenie). - L'Interdiction de SousLouer. Paris, 1930. 1 vol.

WALLING (William English). - Le Mouvement Ouvrier et la Démocratie aux Etats-Unis. Paris, 1930. 1 vol.

\section{REVISTAS}

THE ANNALS OF THE AMERICAN ACADEMY OF POLITICAL AND SOCIAL SCIENCE. - Philadelphia, 1932. 4 vols.

ANNUAIRE DE L'INSTITUT INTERNATIONAL DE DROIT PUBLIC. - Paris, 1931. 2 vols.

ARCHIVES DE PHILOSOPHIE DU DROIT ET DE SOCIOLOGIE JURIDIQUE Nos. 1 e 2. 1931. Paris, 1 vol.

E'CONOMISTE FRANÇAIS. - Journal hebdomadaire. 1932. 52 fasciculos.

JOURNAL DES E'CONOMISTES. - Paris, 1932.8 fa sciculos. 
REVISTA DE DIREITO CIVIL, COMERCIAL e CRIMI-

NAL. - de Bento de Faria. Rio de Janeiro, 12 volumes.

REVUE DES DEUX MONDES. - Paris, 1932. 21 fasciculos.

REVUE DE DROIT INTERNATIONAL PRIVE' - Paris, 1932. 3 volumes.

REVUE D'E'CONOMIE POLITIQUE. - Paris, 1932. 8 fasciculos.

REVUE PHILOSOPHIQUE DE LA FRANCE ET DE, L'E'TRANGER. - Paris, 1932. 3 volumes.

REVUE TRIMESTRIELLE DE DROIT CIVIL - Paris, 1932. 4 volumes.

RIVISTA DI DIRITTO INTERNAZIONALE. - Roma, 1932. 4 volumes.

RIVISTA INTERNAZIONALE DI FILOSOFIA DEL DIRITTO. - Roma, 1932. 6 volumes.

\section{POR PERMUTA:}

\section{OBRAS}

A. D'ESPANET. - Barão do Rio Branco. - Notas politicas e biograficas. 2a. edição. 1 vol.

CARTA QUE O VISORREY DO BRASIL DOM IORGE MASCARENHAS MARQUEZ DE MONTALUÃO ESCREUEU AO EXCELLENTISSIMO CONDE DE NASSAU GENERAL DOS OLÃDESES EM PERNÃBUCO. Lisbôa, 1641.

RELAÇÃO DA CHEGADA QUE TEVE A GENTE DE MATO GROÇO e agora se acha em companhia do senhor D. Antonio Rolim. Lisbôa, 1754. 


\section{REVISTAS}

ACADE'MIE DE DROIT INTERNATIONAL. - Paris. 8 volumes.

ANAIS DA SOCIEDADE JURIDICA SANTO IVO. - Rio de Janeiro, 1932. 1 vol.

ANALES DE LA UNIVERSIDAD DE CHILE. - Chile. 1931. 2 vols.

ANNALES DE L'UNIVERSITE' DE PARIS. - Paris, 1932. 6 volumes.

ANNALI DEL SEMINARIO GIURIDICO DELLA R. UNIVERSITA' DI PALERMO. - Palermo, 1932. 3 vols.

ANNUARIO DELLA REGIA UNIVERSITA' DI CAGLIARI. - Anno Scolastico de 1931-1932. 1 vol.

ANNUARIO DELLA R. U'NIVERSITA' DI TORINO. 1931-1932. 1 vol.

ANNUARIO DELLA R. UNIVERSITA' DEGLI STUDI DI MILANO. - Anno Academico. 1927 a 1930. 3 vols.

ANNUARIO DELLA R. UNIVERSITA' DEGLI STUDI DI PADOVA. - 1926 a 1930.3 vols.

ANNUARIO DELLA R. UNIVERSITA' DI ROMA. 1925 a 1932.5 vols.

ANUARIO DA ESCOLA POLITECNICA DE S. PAULO para o ano de 1932.

ARCHIVOS DE LA UNIVERSIDAD DE BUENOS AIRES. - Buenos Aires, 1931 e 1932.7 vols. 


\section{$-187-$}

ARQUIVOS DA ASSISTENCIA A PSICOPATAS DE PERNAMBUCO. - Recife, 1932. 1 vol.

ARQUIVOS DO INSTITUTO NINA RODRIGUES. - Baía, 1932. 2 vols.

BOLETIM BIBLIOGRAFICO DA BIBLIOTECA DA UNIVERSIDADE DE COIMBRA. - 1927-1928. 3 vols.

BOLETIM DA UNIÃO PAN-AMERICANA. - 1932.6 vols.

BOLETIN DEL INSTITUTO DE CLINICA QUIRURGICA. - Universidad de Buenos Aires, 1932. 1 vol.

BOLETIN DE LA BIBLIOTECA DE LA UNIVERSIDAD DE BUENOS AIRES. 1923. 1 vol.

BOLETIM DO MINISTERIO DA EDUCAÇÃO E SAUDE PUBLICA. - Rio de Janeiro. 1931. 1 vol.

DERECHO Y LEGISLACION. - Revista del Colegio de Abogados del Estado Merida. 1932. 1 vol.

GACETA JURIDICA. - San Cristobal. Venezuela, 1932. 1 vol.

INTERNATIONAL CONCILIATION. - New York, 1932. 11 fasciculos.

JOURNAL DU DROIT INTERNATIONAL. - Paris, 1932. 5 vols.

JURISPRUDENCIA. - Colecion Abadie - Santos. Montevideo, 1932. 1 vol.

JUSTIÇA - (Doutrina - Legislação - Jurisprudençia). Porto Alegre, 1932. 1 vol.

LA GIUSTIZIA PENALE. - Roma, 1932. 13 fasciculos. 
ESPRIT INTERNATIONAL. (L') - Paris, 1932. 1 vol.

MACKENZIE COLLEGE. - S. Paulo, 1932. 2 vols.

NIEMEYERS ZEITSCHRIF FUR INTERNATIONALES RECHT. - 1932. 9 fasciculos.

PARANA' JUDICIARIO. - Doutrina, Jurisprudencia e Legislação. Curitiba, 1932. 2 vols.

REVISTA DA FACULDADE DE DIREITO DA BAI'A. - Baía, 1932. 1 vol.

REVISTA DA UNIVERSIDADE DE MINAS GERAIS. Belo Horizonte, 1930. 1 vol.

REVISTA DE DERECHO Y CIENCIAS SOCIALES. Organo de la Facultad de Derecho y C. Sociales de Asuncion. 1931. 3 vols.

REVISTA DE IDENTIFICACION Y CIENCIAS PENALES DE I.A UNIVERSIDAD DE LA PLATA. - La Plata, 1932.

REVISTA DE JURISPRUDENCIA BRASILEIRA. - Rio de Janeiro, 1932. 9 fasciculos.

REVISTA DE LA UNIVERSIDAD DE BUENOS AIRES. -1931.1 vol.

REVISTA DO INSTITUTO DE ADVOGADOS DE CAMPINAS. - Campinas, 1932. 1 vol.

REVISTA DO INSTITUTO ARQUEOLOGICO E GEOGRAFICO ALAGOANO. vol. XV de 1931.

REVISTA DO INSTITUTO HISTORICO E GEOGRAFICO BRASILEIRO. - Rio de Janeiro, 1931 e 1932.6 vols. 
REVISTA DO INSTITUTO HISTORICO E GEOGRAFICO PARAIBANO. João Pessoa, 1932. 2 vols.

REVISTA DO INSTITUTO ARQUEOLOGICO HISTORICO E GEOGRAFICO PERNAMBUCANO. - Recife, 1932. 3 vols.

REVISTA DO SUPERIOR TRIBUNAL DE JUSTIÇA. - Florianopolis, 1932. 2 fasciculos.

REVISTA DOS CURSOS DA FACULDADE DE MEDICINA DE PORTO ALEGRE. - 1932. 1 vol.

REVISTA JURIDICA. - Orgão oficial do Instituto da Ordem dos Advogados de Pernambuco. Recife, 1931. 1 vol.

REVISTA JURIDICA. - Caracas. Venezuela 5 vols.

REVISTA UNIVERSITARIA. - Organo de la Universidad Menor del Cuzco. 1931. 2 vols.

UNIVERSIDAD DE AREQUIPA. - Arequipa, 1932. 1 vol.

UNIVERSITY OF THE STATE OF NEW YORK. - Bulletin. 1932. 1 vol.

\section{DIVERSOS}

DECISÕES DO SUPERIOR TRIBUNAL DO ESTADO DO RIO GRANDE DO SUL. - Porto Alegre, 1928-1929. 2 vols.

RELATORIO DO TRIBUNAL DA RELAÇÃO DO ESTADO DO RIO DE JANEIRO. - Anos de 1922, 1927, 1928 e 1929, Niteroi. 4 vols. 
UNDEN (Osten). - L'affaire maritime. Upsala. 1932. 1 vol.

UNIVERSITE' DE TOULOUSE. -- La Ville. La Région, 1932. 1 vol.

\section{POR DOAÇÃO: \\ DOAÇÃO dOS AUTORES}

A. DE CAMARGo NEVES. - Comentarios de Politica Economica. Paris, 1921. 1 vol.

A. C. DE SALLES JUNIOR. - Reformas das Tarifas. S. Paulo, 1932.1 vol.

ABELARDO SARAIVA DA CUNHA LOBO. - Curso de Direito Romano. Rio de Janeiro 1931. 3 vols.

A. DE SAMPAIO DORIA. - Principios Constitucionais. S. Paulo, 1926. 1 vol.

ACHILLES LISBOA. - Homenagem do Jardim Botanico. Rio de Janeiro, 1932. 1 vol. á memoria de Goethe.

AFRANIO LAGES. - Do abuso do Direito na execução da promessa de casamento. Maceió, 1932. 1 vol.

ALCANTARA MACHADO. - O exame pericial no Direito Romano. S. Paulo, 1930. 1 Folh.

ALCANTARA MACHADO. - Vida e morte do bandeirante. S. Paulo, 1929. 1 vol.

ALCANTARA MACHADO. - Os Honorarios Medicos. S. Paulo, 1922. 1 vol. 
ALPI (G.). - Carlos Magalhães de Azevedo. Poeta e Umanista Americano. Roma, 1931. 1 Vol.

ALPHEU CANNIÇO. - Sob as arcadas. Cronica de um lustro academico. S. Paulo, 1927. 1 vol.

ALVARO RAMOS DE FREITAS. - Contas assinadas (vendas mercantis) São Paulo, 1932. 1 vol.

ANNIBAL GAMA. - A Conferencia de Washington. Rio de Janeiro, 1924. 1 vol.

ANTONIO DE ALCANTARA MACHADO. - Braz. Bexiga Barra Funda. S. Paulo, 1927. 1 vol.

ANTONIO DE ALCANTARA MACHADO. - Laranja da China. S. Paulo, 1928. 1 vol.

ANTONIO DE ALCANTARA MACHADO. - Pathé Baby. S. Paulo, 1926. 1 vol.

ANTONIO DE ALCANTARA MACHADO. - Anchieta na Capitania de São Vicente. 1929. 1 vol.

ANTONIO M. LEÃO BRUNO. - Contribuição ao tratamento da prenhez extra-uterina. S. Paulo, 1928. 1 vol.

ANTONIO PAULO DA CUNHA - Venda de bens de incapazes. S. Paulo, 1928. 1 vol.

ANTONIO PAULO DA CUNHA. - Preleções das hipotécas sobre as dividas fiscais. S. Paulo, 1931. Broch. 1 vol.

ANTONIO RABBINO. - Oração de formatura pronunciada na solenidade da colação de gráu dos Bachareis de 1932. Rio de Janeiro, 1932. 1 vol.

ARMANDO DE AGUIAR CARDOSO. - A questão da Companhia Telefonica. Rio de Janeiro, 1926. 1 vol. 
ASDRUBAL LINS CALDAS VILLARIM. - Do Ministerio Público em Pernambuco. 1 vol. s/d.

AUGUSTO MEIRA. - Amazonas versus Pará. Belém, 1932. 1 vol.

AURELIANO LEITE. - Memorias de um Revolucionario. S. Paulo, 1932. 1 vol.

BUTLER (Nicholas Murray). - El Mundo Cambiante. Habana, 1932. 1 vol.

CANDIDO DE OLIVEIRA FILHO. - Pratica Civil. Direito das Obrigações. vol. 9. Rio de Janeiro, 1931. 1 vol.

CARLOS ALBERTO A. DE CARVALHO PINTO. - Discurso na colação de gráu dos bacharelandos de 1931 na Faculdade de Direito de S. Paulo, 1 vol.

CINCINATO BRAGA. - Brasil Novo. vol. IV Rio de Janeiro, 1931. 1 vol.

ELIEZER DOS SANTOS SARAIVA. - O Catolicismo Romano ou a velha e fatal ilusão da sociedade. São Paulo, 1932. 1 vol.

FAUSTO DE ALMEIDA PRADO PENTEADO. - Santos Dumont. Sua vida, seus feitos, sua gloria. S. Paulo, 1932. 1 vol.

FONTES JUNIOR. - Catalogo dos discursos e trabalhos parlamentares do Deputado, Senador Estadual e Deputado Federal, Fontes Junior, 1895 a 1930. S. Paulo, Rio de Janeiro, 1 vol.

FRANCISCO FERNANDES SOBRAL. - Repressão juridicosocial ao uso das bebidas alcoolicas. Belo Horizonte, 1931. 1 vol. 
JOÃO ARRUDA: - O Moloch Moderno. Estudos da Crise do Estado. S. Paulo, 1932. 1 vol.

JOÃO DA GAMA CERQUEIRA. - Privilegios de Invenção e Marcas de Fabrica e de Comercio. S. Paulo, 1930. 2 vols.

JORGE AMERICANO. - Da Ação Rescisoria dos julgados no Direito Brasileiro, 2a. edição. S. Paulo, 1926. 1 vol.

JORGE AMERICANO. - Da Ação Pauliana. S. Paulo, 1932. 1 vol.

JORGE AMERICANO. - Aplicação do Direito. Rio de Janeiro, 1930. 1 vol.

JORGE AMERICANO. - Do Abuso do Direito no exercicio da demanda. 2a. edição. S'. Paulo, 1932. 1 voll.

JORGE AMERICANO. - Dos direitos que se exteriorisam pela posse. Do compromisso de venda de imoveis. S. Paulo, 1926. 1 vol.

JOSE' CARLOS DE MACEDO SOARES. - Brasil and the League of Nations. Paris, 1928. 1 vol.

JOSE' DE MESQUITA. - Atentado contra a Justiça. A redução dos vencimentos da magistratura. Cuiabá, 1932. 1 vol.

JOSE' NIEPCE DA SILVA. - As vias estrategicas para as tronteiras meridionais. S. Paulo, 1930. 1 vol.

LAURINDO LEÃO. - Historia da Filosofia 1929-1930. 2 vols.

LEOPOLDINO AMARAL MEIRA. - Estudos da Posse e das Ações Possessorias segundo o regimen do Codigo Civil Brasileiro. S. Paulo, 1928. 1 vol. 
LEVEN VAMPRE' - S. Paulo, terra conquistada. S. Paulo, 1932. 1 vol.

LOPES GONÇALVES. - Folha corrida. Rio de Janeiro, 1931. 1 vol.

LUCIO JOSE' DOS SANTOS. - Missão Universitaria nos Estados Unidos. Belo Horizonte, 1930. 1 vol.

MANOEL LACERDA. - Processo Crime. Uberlandia. 1932. 1 vol.

MANUEL VIOTTI. - Dactyloscopie et Anthropologie. Lyon, 1931. 1 vol.

MARIO DE ANDRADE. - Ensaio sobre a Musica Brasileira. S. Paulo, 1928. 1 vol.

MIGUEL COUTO. - No B'rasil só ha um problema nacional: a educação do povo. (Conferencia.). Rio de Janeiro, 1927,1 vol.

NAPOLEÃO LOPES. - Sociedade contra sociedade. 4a. edição. S. Paulo, 1932. 1 vol.

NíCOLAO DUARTE SILVA. - Libero Badaró, 1830-1930. S. Paulo, 1931. 1 vol.

NOE' AZEVEDO. - Da Prova na Investigação da Paternidade. S. Paulo, 1928. 1 vol.

NOE’ AZEVEDO. - Ação Revocatoria. S. Paulo, 1932.1 vol.

OLYNTHO NOGUEIRA. - A Empirica Policia atual e a Policia Tecnica de amanhã. Rio de Janeiro. 1 vol. s. d.

ORGAZ (Alfredo). - Responsabilidad por el Hecho de las cosas inanimadas. Legislacion Argentina y Comparada. Cordoba, 1931. 1 vol. 
PAIS BARRETTO FILHO. - Notas da Promotoria Criminal - Vitoria. 1 vol.

PAULO DUARTE. - Que é que ha? S. Paulo, 1931. 1 vol.

PAULO DUARTE. - Agóra nós. S. Paulo, 1927. 1 vol.

PAULO PRADO. - Paulistica. Historia de S. Paulo. S. Paulo, 1925. 1 vol.

PAULO PRADO. - Retrato do Brasil. IVa. edição. Rio de Janeiro, 1931. 1 vol.

REA (George Brousson). - «The Open Door». 1 vol.

RIVAROLA (Rodolfo). - L'Université Sociale. Traduit de l'espagnol para Camille Morel. Paris. 1 vol.

RODRIGO OCTAVIO. - Les Sauvages Américains devant le Droit. Paris, 1931. 1 vol.

RODRIGO OCTAVIO. - Alexandre de Gusmão et le sentiment américain dans la Politique Internationale. Paris, 1930. 1 vol.

RODRIGUES DORIA. - Epilepsia e epilepticos notaveis. Baía, 1932. 1 vol.

SERGIO MILLIET - Terminus sêco e outros cocktails. - S. Paulo, 1932. 1 vol.

SOLAR (Domingos A.) - José Toribio Medina. Chile, 1932. $1 \mathrm{vol}$.

SOARES DE FARIA. - Investigação da Paternidade ilegitima. S. Paulo, 1926. 1 vol.

SOARES DE FARIA. - Da exlusão de socios nas sociedades de responsabilidade ilimitada. S. Paulo, 1926. 1 vol. 
SOARES DE FARIA. - Da Concordata Preventiva da Falencia. S. Paulo, 1932. 1 vol.

THO’T (Ladislau). - A Evolução Historica do Direito Penal. Separata do Boletim do Instituto de Criminologia de Lisbôa. Lisbôa, 1932. 1 vol.

THO'T (Ladislau). - Il problema sessuale nelle carceri. Roma, 1931. 1 vol.

VILLANOVA (Antonio Royo). - Eltmentos de Derecho Administrativo. Madrid, 1929. 1 vol.

VIRGILIO DE BARROS. - Embargos. Manaus. 1 vol. s/d.

WALDEMAR FERREIRA. - O Conhecimento do Transporte Ferroviario. S. Paulo, 1931. 1 vol.

\section{DOAÇÃO dA “THE CARNEGIE ENDOWMEAT FOR INTERNATIONAL PEACE"}

ANZILOTTI (Dionisio). - Cours de Droit International. Paris, 1929. 1 vol.

POLITIS (Nicolas). - The New Aspects of International law. Washington, 1928. 1 vol.

SCOTT (James Brown). - The Hague Court Report. New York, 1932. 1 vol.

SCOTT (James Brown). - The Treaties of 1778. Baltimore 1928. 1 vol.

SCOTT (Samuel Brown). - The Classics of International Law., 1927. 2 vols. 
WEHBERG (Hans). - The Outlawry of War. Washington, 1931. 1 vol.

AMERICAN JOURNAL OF INTERNATIONAL LAW. vol. 23. April, 1929. Washington.

CARNEGIE ENDOWMENT FOR INTERNATIONAL PEACE. - Year Book. 1927 a 1931. Washington, 1931. 5 vols.

COLLECTION OF NATIONALITY LAWS. - New York. 1929. 1 vol.

TREATIES AND AGREEMENTS WITH AND CONCERNING CHINA. - 1919-1929. Washington, 1929. 1 vol.

SMITH COLLEGE STUDIES IN SOCIAL WORK. - 1931. 3 vols.

\section{DOAÇÃO DO DR. COUTO DE BARROS}

BAINVILLE (Jacques.) - Heur et Malheur des Français. Paris, 1924. 1 vol.

HESNARD (A.). - La Relativité de la Conscience de soi. Introduction à la Psychologie Clinique. Paris, 1930. 1 vol.

NORDMANN (Chatles). - Notre Maître le Temps. Paris, 1929. 1 vol.

POUQUET (Jeannr Maurice). - Le Salon de Madame Armand de Caillavet. Paris, 1 vol.

\section{DOAÇÃO DO DR. JOSE' CARLOS DE MACEDO SOARES.}

\section{OBRAS}

ALBERTO CARLOS DE MENEZES. - Pratica dos Tombos e e segunda parte anexa aos Juizes Divisorios. Lisbôa, 1886. 1 vol. 
ANDRE' REBOUÇAS. - A Seca nas Provincias do Norte. Rio de Janeiro, 1877. 1 vol.

B. DO CARMO BRAGA JUNIOR. - Letra de Cambio. Nota Promissoria. Cheque. Titulos ao Portador. Rio de Janeiro - Porto, 1914, 1 vol.

BENJAMIN DO CARMO BRAGA JUNIOR. - Regimem das Marcas de Fabrica e de Comercio. Rio de Janeiro, 1922. 1 vol.

BIBLIOTECA DELL'ECONOMISTA. - Torino, 1850 a 1908. 64 vols.

BILLAUDANT (Maurice). - La Politique Monétaire de l'Allemagne depuis la fin de la guerre. Paris, 1926. 1 vol.

BLANCO CONSTAUS (Francisco). - Estudios Elementares de Derecho Mercantil. Tomo I e II. Madrid, 1901.

BRIEY (R. de). - Essai sur l'Association du Capital et du Travail par l'Actionnariat Ouvrier. Bruxelles. Paris, 1914. 1 vol.

BUFNOIR (C.). - Code Civil Allemand. Paris, 1932. 1 vol.

BUTLER (Geotrey). - A Handbook to the League of Nations. New York. Toronto, 1925. 1 vol.

CANDIDO COSTA. - Coletaneas de Legislação Federal. Manáus, 1905. 1 vol.

CARLOS PORTO CARREIRO. - Lições de Economia Politica e Noções de Finanças. Rio de Janeiro, 1931. 1 vol.

CARNEIRO MAIA. - Codigos dos Municipios do Estado de São Paulo, 1929. 1 vol. 
CERESETO (G. B.). - Le case popolari. Leggi. Torino, 1904. 1 vol.

CHAMBERLAIN (Lawrence). - The Principles of Bond Investment. New York, 1913. 1 vol.

CLAMAgERAU (J. J.). - Etudes Politiqués. Paris, 1904. 1 vol.

CODE DE PROCEDURE CRIMINELLE ESPAGNOL. Traduit et annoté para Gabriel Verdier, Paris, 1898. 1 vol.

CODERCH (Rafael). - Retiros Obreros. Madrid, 1919. 1 vol.

CODIGO CIVIL DE LA REPUBLICA ORIENTAL DE URUGUAY - Madrid, 1929. 1 vol.

CODIGO DE COMERCIO DE LA REPUBLICA ARGENTINA Y LEYS COMPLEMENTARIAS. - Buenos Aires. 1 vol.

CODIGO RURAL DE LA REPUBLICA ORIENTAL DE URUGUAY. - Montividéo, 1918. 1 vol.

COSTAMAgNA (Carlos). - Manuale di Diritto Corporativo Italiano. Torino, 1927. 1 vol.

CROISET (S. J. ). - ANO CRISTÃO OU DICIONARIO PARA TODOS OS DIAS DO ANO. Vols. I e II. Portugal. Brasil.

CROSSARA (Aldo). - Íl Deprezzamento come equilibrio di costi. Padova. 1 vol. sid.

ECONOMIA SOCIAL. - Situação do operariado. Lisbôa, 1904. 1 vol

FELIX (M. Maurice). - Petit Dictionnaire de Droit Municipai. Paris, 1 vol. 
GALLIARD (G. Olphe). - Histoire Economique et Financière de la Guerre 1914-1918. 1 vol.

GAMBARDELLA (Adolfo). - Formazione e variazione di Capitale nelle Societá Anonime. Milano, 1 vol. s/d.

GARRAUD (R.). - Précis de Droit Criminel.- Paris, 1921. 1 vol.

GAUTIER (J.) - Bibliographie des Sciences Economiques, Politiques et Sociales. 1909. 1 vol.

GILLIARD (Pierre). - Le tragique destin de Nicolas II et de sa famille. Paris, 1921. 1 vol.

GRIZIOTTI (Benevenuto). -- La politica finanziaria italiana. Milano, 1926. 1 vol.

HANSEN (Emilio). - La Moneda Argentina. Buenos Aires, 1916. 1 vol.

HARISTOY (Just.). - L'Impôt sur le Revenu. Paris, 1910. 1 vol.

JEZE (Gaston). - Cours de Finances Publiques. Paris, 19261927. 1 vol.

JOAQUIM JOSE' CAETANO PEREIRA E SOUZA. Primeiras linhas sôbre o Processo Civil. Rio de Janeiro, 1906. 1 vol.

JOLLET (Roger). - La Stabilisation du Change Italien. Paris, 1926. 1 vol.

JOSE' TAVARES BASTOS. - Serviço Policial do Estado do Rio de Janeiro. Paris, 1904. 1 vol. 
JOSE' ULPIANO PINTO DE SOUZA. - Das Clausulas Restritivas da Propriedade. S. Paulo, 1910. 1 vol.

LA SERNA (M. de). - Dictionnaire Bibliographique du Quinziéme Siècle. Bruxelles, 1805-1806-1807. 3 vols.

LUIZ DOS SANTOS VILHENA. - Cartas de Vilhena. Noticias Soteropolitanas e Brasilicas. Baía, 1922. 1 vol.

LAURENCE (Fernand). - La Stabilisation du Franc. Paris, 1926. 1 vol.

LESCURE (Jean). - Des Crises Générales et Périodiques de Surproduction. Paris, 1910. 1 vol.

LOPES DA CRUZ. - Da Etica das Leis Civis e Politicas do Brasil. Rio de Janeiro, $19271 \mathrm{vol}$.

LABORDE - LACOSTE - CRE'MIEU ET BONNECARRE'RE. - Précis de Droit Civil. Paris, 1926-1927 2 vols.

LOUGEVIALLE (Bernard de). - Vers la monnaie saine. Paris. 1 vol. s/d.

MARGARINO TORRES. - Nota Promissoria. Rio de Janeiro, 1917. 1 vol. s/d.

MARTECCHINE (Antonio). - Codice Civile Generale pel Principato del Montenegro. Spalato, 1900. 1 vol.

MARTINET (C.). - Code Pénal Hongrois des Contraventions, Paris, 1885, 1 vol.

MINCER (Tadeus). - Zloty Polonais. La Réforme monétaire en Pologne et ses suites. Paris. 1 vol. 
ORMAZA (Joaquim Mendez). - La Question Social en sus relaciones con los distintos modernos sistemas de pago de salarios Madrid. 1 vol.

PAOU (Marcel). - L'Immigration en France. Paris, 1926. 1 vol.

PAULO MARIA DE LACERDA. - Do Cheque no Direito Brasileiro. Rio de Janeiro. 1 vol. s/d.

PENA (José B.). - Deuda Argentina. - Compilation de leys, decretos, resoluciones, notas y contratos. Buenos Aires, 1907. 2 vols.

PEREIRA (Huarcar). - Appunti sui Legnami dello Stato di San Paolo. San Paolo, 1910. 1 vol.

PETRINI-PAUL (Pierre). - L'Insécurité dans les Transactions Immobiliêres. Paris, 1922. 1 vol.

PLASSARD (Jean). - Evolution de la nature Juridique des attentats á la sureté exterieure dee l'Etat. Paris, 1924. 1 vol.

PONTES DE MIRANDA. - Do Direito das Obrigações. - Rio de Janeiro, 19171 vol.

PUPIN (René). - La Richesse de la France devant la guerre. Paris, 1916. 1 vol.

QUESADA (Sixto J.). - Las Finanzas de la Republica Argentina. Buenos Aires, 1892. 1 vol.

QUESADA (Vicente J.). - Historia Diplomatica LatinoAmericana. Buenos Aires, 1918. 3 vols.

RAMALHO ORTIGÃO. - O ano comercial economico e financeiro de 1917. Rio de Janeiro 1918. 1 vol.

RENAULT (Carlos). - Las Huelgas. Madrid, 1 vol. s/d. 
ROBERT (Jean Maxime). - Dépréciation de la Monnaie et Equilibre Budgétaire. Paris, 1922-1923. 1 vol.

RODRIGO OCTAVIO - Letra de Cambio e Nota Promissoria. Rio de Janeiro, 1911. 1 vol.

ROLDAU (Alcibiades). - Elementos de Derecho Constitucional de Chile. Santiago de Chile, 1913. 1 vol.

ROUX (J A.). - Cours de Droit Pénal et de Procédure Pénale. Paris, 1920. 1 vol.

SPENGLER (Oswald). - La Decadencia de Occidente. Calpe, 1926-1927. 2 vols.

TIZAC (G. d'Ardenne de). - Droit D'Auteur. Lois et Régléments. Paris, 1913. 1 vol.

TREZZI (Giuseppe). - La Posizione giuridica della Santa Sede nel Diritto Internazionale. Roma, 1929. 1 vol.

ULLMANN (Emanuele). - Trattato di Diritto Internazionale Publico. Torino, 1914. 1 vol.

WILliaMS (Ivy). - The Swiss Civil Code. 1925. 1 vol.

WILSON (Woodrow). - Mensagens, Alocuções e Discursos. Rio de Janeiro, 1918. 1 vol.

ZACARIAS DE LIMA. - Os problemas do Café. Monte Alto, 1929. 1 vol.

ZEBALLOS (Estanisláo S.). - Cuestiones y Legislacion del Trabajo. Buenos Aires, 1919. 1 vol. 


\section{REVISTAS}

BOLETIM DA COMISSÃO GEOGRAFICA E GEOLOGICA DO ESTADO DE SÃO PAULO, de 1890 a 1902.2 vols.

REVUE GENERALE DE DROIT INTERNATIONAL PUBLIC. Paris, 1894 a 1923. 30 vols.

\section{DIVERSOS}

ALBERTO VEIGA. - O Declive. Santos, 1921. 1 vol.

AZEVEDO SODRE' - Oito mêses de administração interina. Rio de Janeiro, 1917. 1 vol.

BEN'TO JORDÃO DE SOUZA. - Atos no Juizo de Ausentes e de Orfãos. S, Paulo. 1922. 1 vol.

CATALOGO GERAL DA BIBI.IOTECA DA SOCIEDADE HUMANITARIA DOS EMPREGADOS NO COMERCIO DE SANTOS. - Santos, 1927. 1 vol.

CONGRESSOS DE ESCOTEIROS NO BRASIL. (1922-1923). Rio de Janeiro. 1 vol.

DOCUMENTOS PARLAMENTARES. - 1892-1900. Rio de Janeiro, 1913. 18 volumes.

INDICADOR COMERCIAL PAULISTA PARA 1914-1915. 4 fasciculos.

L'ETAT DU PARA' — Le Brésil à Turim. 1911. 1 vol.

MANUAL DOS BANCOS NO BRASIL. - Registrado na Biblioteca Nacional. Rio de Janeiro, 1928. 1 vol.

MENSAGENS APRESENTADAS A' ASSEMBLEA LEGISLATIVA. - pelo Snr. Manoel Correa Dantas. Pressidente do Estado de Sergipe. Aracajú 19"27-1930. 
OSCAR MARCONDES DE SOUZA. - O Estado de S. Paulo, Fisico, Politico, Economico e Administrativo. S. Paulo, 1915. 1 vol.

PROBLEME DU CHOMAGE EN GRANDE-BRETAGNE. (Le). - Genéve, 1924. 1 vol.

REGULAMENTO DO IMPOSTO DE CONSUMO. - Rio de Janeiro, 1921. 1 vol.

RELATORIO DO BANCO DO BRASIL. - Rio de Janeiro, 1917. 1 vol.

RELATORIO DA SECRETARIA DOS NEGOCIOS DO INTERIOR E DA JUSTIÇA. - S. Paulo, 1905. 1 vol.

RELATORIO DA ASSOCIAÇÃO COMERCIAL DE S. PAULO. - S. Paulo, 1920. 1 vol.

SOCIETE' DES NATIONS - Indications Relatives a L'Etat des Armements des divers Pays. Communication du Gouvernement Brésilien. Genève, 1932. 1 folh.

\section{DOAÇÃO DO DR. SERGIO MILLIET}

\section{OBRAS}

ALBERTO LAMEGO. - A Terra Goytacá. Paris, 1913. 1 vol.

BAPTISTA PEREIRA. - Civilisação contra Barbarie. S. Paulo, 1928. 1 vol.

BAPTISTA PEREIRA. - O Brasil e a Raça. S. Paulo, 1928. 1 vol.

CLEMENCEAU (George). - Grandeurs et misères d'une victoire. Paris, 1930.1 vol. 
EUGENIO DE CASTRO. - Diario de Navegação 1530-1532. vols. $1 .^{\circ}$ e $2 .^{\circ}$. Rio de Janeiro, 1927.

FREUD (Sigmund). - Trois essais sur la théorie de la sexualité. Paris, 1924. 1 vol.

FREUD (Sigmund). - Psychopathologie de la vie quotidienne. Paris, 1926. 1 vol.

GRAÇA ARANHA. - Machado de Assis e Joaquim Nabuco. — São Paulo, 1923. 1 vol.

GEORGE (Henry). - Progrés et Pauvreté. Traduit de l'anglais par P. L. Le Monnier. Paris, 1925. 1 vol.

HESNARD et R. LAFORGUE. - Les Processus d'AutoPunition. Paris, 1931. 1 vol.

J CAPISTRANO DE ABREU. - Um visitador do Santo Oficio á cidade do Sálvador da Baía de todos os Santos, 1591-1592. Rio de Janeiro, 1922. 1 vol.

LUDWIG (Emil). — Bismarck. Paris, 1929. 1 vol.

MARTIM FRANCISCO. - Viajando. S. Paulo, 1929. 2 vols.

MARTIM FRANCISCO.-Contribuindo. S. Paulo, 1921.1 vol.

POTTER (C. F.). - Les Fondateurs de Religions. Paris, 1930. 1 vol.

SEILLIERE (Ernest.) - Marcel Proust. Paris, 1932. 1 vol.

SUD MENNUCI. - A Crise Brasileira de Educação S. Paulo, 1930. 1 vol.

VIVALDO COARACY. - O Caso de S. Paulo. S. Paulo, 1931. 1 vol. 


\section{REVISTAS}

LE CRAPOUILLOT. - Numéro spécial sur L'Amérique et les Américains, Paris, 1930. 1 vol.

LE MOIS. Paris, 1932. 10 vols.

REVUE FRANÇAISE DE PSYCHANALYSE. - Paris, 1932. 1 vol.

REVUE METAPSYCHIQUE. - Paris, 1930. 1 vol.

\section{DIVERSOS}

BRAZILIAN COTTON. - Manchester, 1931. 1 vol.

\section{DOAÇÃO DO DR. SPENCER VAMPRE'}

\section{OBRAS}

ALBERTO LAMEGO. - A terra Goyatacá. Paris, 1913. 1 vol.

ALBERTO DE MORAIS CARVALHO. - Apreciação filosofica Juridica e Analitica das principais alterações feitas pelo Codigo Civil Português. Lisbôa, 1871. 1 vol.

ART (George): - Pour développer notre mémoire. Paris, 1 vol.

BARCA (Pedro Calderon de la). - Das Grobe Welttheater. 1 vol.

BIRE' (Edmond). — Les Défenseurs de Louis XVI. Lyon, 1896. 1 vol.

COMTE DE CAYLUS. - Oeuvres badines et galantes. Paris, 1920.

GOURMONT (Rémy de). - Physique de l'Amour. Paris. 1922. 1 vol. 
J. M. MAC-DOWELL DA COSTA. - Apelação Civil N. 6295. 1 voi.

JOÃO JOSE' RODRIGUES. - Miscelanea Juridica. Rio de Janeiro, 1875. 1 vol.

JULIEN (Félix). - Papes et Sultans. Paris, 1879. 1 vol.

LEONARDO PINTO. - Pseudo estelionato. S. Paulo, 1932. 1 vol.

LOPEZ (VICENTE FIDEL) - Manual de la Historia Argentina. Buenos Aires, 1916.1 vol.

LUIS CAETANO FERRAZ. - Compendios dos minerais do Brasil em forma de Dicionario. Rio de Janeiro, 1929. 1 vol.

MARIO DE ANDRADE RAMOS. - Governo da Moeda. Questões Sociais. 1 vol.

MOREIR. DE AZEVEDO. - Homens do passado. Rio de Janeiro. 1875. 1 vol.

MUCIO CAMPOS. - Ação de indenização de um locador de serviços. 1 vol. $\mathrm{s} / \mathrm{d}$.

NERVA (Emile). - Philosophie Positive de l'Histoire, 1878. 1 vol.

NEFZAONI (Cheikh). - Le Livre d'Amour de l'Orient. Paris, 1922. 1 vol.

NOE' DE AZEVEDO e VICENTE FERRAZ PACHECO. - Apelação N. 16.710. Rio Claro. 1930. 1 voì.

PANNIER (George). - Société d'Assurance sur la Vie. Paris, 1905. 1 vol. 
SAGERET (Emile). - Essais de Philosophie Synthetique. Paris, 1928. 1 vol.

SORIAU (Paul). - L'Entrainement au Courage. Paris, 1926. 1 vol.

STEINER (Rudolf). - La Philosophie de la Liberté. Paris, 1923. 1 vol.

SAMUEL BACCARAT. - Um caso de violação de segredo profissional. Santos, 1931. 1 vol.

VINOGRADOFF (Paul). - Principes Historiques du Droit, Paris, 1924. 1 vol.

\section{REVISTAS}

ANAIS DA FACULDADE DE MEDICINA DE S. PAULO. - 1931. 1 vol.

BOLETIM DO INSTITUTO DA ORDEM DOS ADVOGADOS BRASILEIROS. - Rio de Janeiro, 1930. 2 vols.

REVISTA DO INSTITUTO HISTORICO E GEOGRAFICO DE S. PAULO. - 1930. S. Paulo, 1931.

\section{DIVERSOS}

ACRE SETENTRIONAL. - Polemica entre Rui Barbosa e Gumercindo Bessa. 1 vol.

COMPTE RENDU COMPLET DES TRAVAUX DU CONGRES MAÇONIQUE SPIRITUALISTE. - Paris, 1908. 1 vol.

CONFERENCIAS E PRINCIPAIS TRABALHOS DO ANO DE 1910. INSTITUTO DA ORDEM DOS ADVOGADOS BRASILEIROS. - Rio de Janeiro, 1912. 1 vol. 
ESTATUTOS DA LIGA DE DEFEZA NACIONAL. - Rio de Janeiro, 1924. 1 vol.

RELATORIO DA ASSOCIAÇÃO COMERCIAL DO RIO DE JANEIRO. — Rio 1924, 1926, 1927, 1928 e 1930. 5v ols.

SATZUNGEN DER GESELLSCHAFT GERMANIA. S. Paulo. 1 vol.

XAVIER DE OLIVEIRA. - Intercambio Intelectual Americano. Rio de Janeiro, 1930. 1 vol.

\section{DOAÇÃO dE DIVERSOS}

\section{OBRAS}

A. VIEIRA MARCONDES. - Consideração sobre a nocividade da industria de trapos em S. Paulo. 1919. 1 vol.

ADELINO LEAL. - Estudo Fisico-Clinico da Essencia do Chenopodium Ambrosiaides. S. Paulo, 1920. 1 voi.

ALVARO SANCHES. - Dois anos de trabalho na nova Delegacia de Saude de S. Carlos e seus resultados. S. Paulo, 1920. 1 vol.

ASPETOS DA ECONOMIA RURAL BRASILEIRA. - Rio de Janeiro, 1922. 1 vol.

AURELIO TEIXEIRA DE CARVALHO. - A luta cntra ao Anquilostomose no primeiro distrito Sanitario de Santos. S. Paulo, 1920. 1 vol.

BERNARD (André). - Du Contrat de Marché. Paris, 1897. 1 vol.

BOM (Maurice J.). - La Crise Américaine Paris. 1 vol. s/d. BURCKHARDT் (A.). - La grammaire de la Banque. 1 vol. 
CARLOS LUIZ MEYER e JOAQUIM RABELLO TEIXEIRA. - A Grippe Epidemica no Brasil e especialmente em S. Paulo. 1920. 1 vol.

CLOVIS MOREIRA SPINOLA. - «Etica Profissional». 1 folheto. s/d.

DIOGO DE FARIA. - Os inimigos dos nossos livros. S. Paulo, 1919. 1 vol.

ENJOLRAS VAMPRE' - Contribuição ao mal de engasgo. S. Paulo, 1919. 1 vol.

ENJOLRAS VAMPRE' - Uma Epidemia de Polinevrites Arsenicaes. S. Paulo, 1920. 1 vol.

FALCÃO DE LAACERDA (Arthur de Barros). - Exposição dos Direitos do Banco Evolucionista sobre 25.000 hectares de terras que comprou á Nação, situadas no Municipio da Capital do Estado de S. Paulo. 1921. 1 vol.

FERNANDO MAGALHÃES. - O Centenario da Faculdade de Medicina do Rio de Janeiro, 1832-1932. 1 vol.

GROTEURS (Hugo). - Annual Lecture. London, 1930. 1 vol.

GUERNER (Nuno). - Profilaxia das verminoses em S. Bernardo. S. Paulo, 1920. 1 vol.

GUILHERME ALVARO. - A campanha sanitaria de Santos - suas causas e seus efeitos. S. Paulo, 1921. 1 vol.

H. CESAR DIOGO. - Profilaxia da Opilação. S. Paulo, 1920. 1 vol.

HANDELMANN (H.). - HISTORIA DO BRASIL. Rio de Janeiro, 1931. 1 vol. 
JOÃO BAPTISTA DA ROCHA. - Estudos das aguas do Estado de S. Paulo. S. Paulo e Rio, 1920. 1 vol.

MANOEL GODOFREDO DE ALENCASTRO AUTRAN. Novo Roteiro dos Orfãos. Rio de Janeiro. - Paris. 1 vol.

MOYANO (Juan Agustin). - Efetos de las nulidades de los. Atos Juridicos. Buenos Aires, 1932. 1 vol.

OCTAVIO MARCONDES MACHADO. - Epidemia de Impaludismo na Usina Ester e Cosmopolis e sua protilaxia. S. Paulo, 1919. 1 vol.

SALLES GOMES FILHO. - Epidemia de Poliomielite infantil em Vila Americana. S. Paulo, 1919. 1 vol.

SALLES GOMES FILHO. - Profilaxia do impaludismo em Vila Americana - Nova Odessa. - Carioba e Salto Grande. S. Paulo, 1919. 1 vol.

SALLES GOMES FILHO. - Profilaxia da Sifilis em S. Paulo. 1920.1 vol.

THAUMATURGO AZEVEDO. - O Acre-Limites com a Bolivia. Rio de Janeiro, 1901. 1 vol.

WALDEMAR DE OLIVEIRA. - Profilaxia da febre amarella no Estado de S. Paulo. 1 vol.

\section{REVista E JORNAIS}

A CHAVE. - (Jornal Academico). - 1 vol. Doação do Dr. Paulo Duarte.

ATAS DA SEGUNDA CONFERENCIA LATINO-AMERICANA DE NEUROLOGIA, PSIQUIATRIA E MEDICINA LEGAL. - S. Paulo. Brasil. 1 vol. 
ANNALES DU MINISTERE DE L'AGRICULTURE. Paris, 1930. 1 vol.

ANAIS DA CAMARA MUNICIPAL DE SÃO PAULO. 1927. São Paulo, 1 vol.

ANAIS DO MUSEU PAULISTA. - Tomos IV e V S. Paulo, 1931. 2 vols.

ANAIS DA ESCOLA DE MINAS DE OURO PRETO. N. 22.1931 .1 vol.

ANNUAIRE DE LA PRESSE FRANÇAISE ET ETRANGERE ET DU MONDE POLITIQUE. - Paris. Bruxelles. Londres, 1926. 1 vol.

ANNUAIRE STATISTIQUE INTERNATIONAL. - Société des Nations Genéve, 1924, 1926, 1927 e 1928. 3 vols.

ANUARIO DA FACULDADE DE DIREITO DE SÃO PAULO. - S. Paulo, 1932. 1 vol.

ANUARIO DE LA DIRECION G. DEL REGISTRO DEL. ESTADO CIVIL. - Montevideo, 1932. - 1 vol.

ANUARIO DEMOGRAFICO. - Seção de Estatistica Demografico-Sanitaria. Ano de $1928.10^{\circ}$ e $2 .^{\circ}$ Vols. S. Paulo, 1929. 2 vols.

ANUARIO DO ENSINO DO ESTADO DE S. PAULO. 1914. 1 vo!.

ANUARIO ESTADISTICO DE LA REPUBLICA ORIENTAL DEL URUGUAY. - Montevideo, 1932. 4 vols.

ANUARIO ESTATISTICO DO CEARA' - Fortaleza, 1931. 1 vol.

ARQUIVO NACIONAL (Publicações). - Rio de Janeiro, 1919, 1922 e 1929. 3 vols. 
ATLANTIDA. - Mensario Artistico, Literario e Social para Portugal e Brasil. Nos. 1, 3, 4, 5, - Lisbôa. 4 vols.

BOLETIM DA REVISTA DO INSTITUTO HISTORICO E GFOGRAFICO BRASILEIRO. - Rio de Janeiro, 1930, 1931 e 1932.6 vols.

BOLETIM DE ARIEL. - Rio de Janeiro. 11 fasciculos.

BOLETIM DE EUGENIA. - Rio de Janeiro, 1929, 1930 e 1931. 1 vol.

BOLETIN DE LA BIBLIOTECA «AMERICA DE LA UNIVERSIDAD DE SANTIAGO DE COMPOSTELA. 1929. 1 vol.

BOLETIN DE LA OFICINA SANITARIA PANAMERICANA. - 1932. 3 vols.

BOLETIN DO DEPARTAMENTO DE TRABALHO AGRICOLA. - S. Paulo, 1932. 2 fasciculos.

BOLETIM DO INSTITUTO DE ENGENHARIA. - S. Paulo, 1932. 6 vols.

BOLETIM DO MUSEU NACIONAL. - Rio de Janeiro, 1930. 1 vol.

BOLETIN MENSUAL DE LA SOCIEDAD DE LAS NACIONES. 1932. 1 vol.

CERVANTES. - Revista Mensual Ilustrada. - Habana. 1932. 2 fasciculos.

CORREIO DA TARDE. - S. Paulo, 1932.

DIARIO DA NOITE. - São Paulo, 1932.

DIARIO DE SÃO PAULO. — S. Paulo, 1932. 
DIARIO NACIONAL. - S. Paulo, 1932.

DIARIO OFICIAL DO ESTADO DE S. PAULO. - S. Paulo 1932.2 vols.

DIARIO OFICIAL DOS ESTADOS UNIDOS DO BRASIL. - Rio de Janeiro, 1932. 2 vols.

DIARIO POPULAR. - S. Paulo, 1932.

EDUCAÇÃO EUGENICA. - Rio de Janeiro, 1932. 1 vol.

ESTADO DE SÃO PAULO (O.). - S. Paulo, 1932.

FOLHA DA MANHÃ (A). - S. Paulo, 1932.

FOLHA DA NOITE (A). - S. Paulo, 1932.

GAZETA (A) - S. Paulo, 1932.

GIORNALE DI POLITICA E DI LETTERATURA. - Roma, 1932. 5 vols.

GLOBO (O). — Rio de Janeiro, 1932.

JORNAL (O). - Rio de Janeiro, 1932.

JORNAL DO BRASIL (O). - Rio de Janeiro, 1932.

JORNAL DO COMERCIO (O). - Rio de Janeiro. 1932.

LITERATURA ARGENTINA. - Bibliografia General Argentina. Buenos Aires, 1929. 6 tasciculos.

NOSSA REVISTA. - Organização e Cultura. 1932. 4 fasciculos.

O XI DE AGOSTO. - S. Paulo, 1932. 2 vols.

PLATEA (A). - S. Paulo, 1932. 
PUBLICAÇÕES DO ARQUIVO NACIONAL. - Rio de Janeiro, 1931. 3 vols.

RAZÃO (A). - S. Paulo, 1932.

RESENHA JUDICIARIA. - Publicação trimestral de Doutrina, Jurisprudencia e Legislação. Espirito Santo, 1919. 2 vols.

REVISTA DE DERECHO CIVIL. - Buenos Aires, 1931. 1 vol.

REVISTA DO INSTITUTO DE CAFE' DO ESTADO DE SÃO PAULO. - 1932. 8 vols.

REVISTA DOS TRIBUNAIS. - S. Paulo, 1932. 6 vols.

REVISTA ECONOMICA SUDAMERICANA. - Montevidéo. 1931. 2 fasciculos.

REVISTA MEDICA DE PERNAMBUCO. - Recife, 1932. 1 vol.

REVISTA NACIONAL DE EDUCAÇÃO. - Rio de Janeiro, 1932. 1 vol.

REVISTA NOVA. - S. Paulo, 1932. 1 vol.

TIRO DE GUERRA (O). - Revista de Assuntos Militares. 1931. 1 fasciculo.

\section{DIVERSOS}

ATAS DA CAMARA DA VILA DE S. PAULO. - de 1562 a 1743. S. Paulo, 1914-1916. 12 vols.

ALMANAQUE AGRICOLA BRASILEIRO. - S. Paulo, 1917. 1 vol. 
ANNAIS DO VIII CONGRESSO BRASILEIRO DE MEDICINA. Rio de Janeiro, 1925. 1 vol.

ARQUIVO NACIONAL DOS ESTADOS UNIDOS DO BRASIL. - Relatorio anual do Diretor referente a 1930. Rio de Janeiro, 1931. 1 vol.

CENTENARIO DA ACADEMIA NACIONAL DE MEDICINA. - VI conferencia Sul-Americana de hygiene, Microbiologia e Patologia. Rio de Janeiro, 1931. 1 vol.

CONGRESSO DE NEUROLOGIA PSIQUIATRA E MEDICINA LEGAL. - Rio de Janeiro, 1932. 1 vol.

CONGRESSO BRASILEIRO DE EUGENIA. - Atas e trabalhos. Rio de Janeiro, 1929. 1 vol.

DIARIO DE SECIONES DE LA CAMARA DE SENADORES. - Buenos Aires, 1910-1912. 2 vols.

DIARIO DE SECIONES DE LA CAMARA DE SENADORES DE LA REPUBLICA ORIENTAL DEL URUGUAY - Montevidéo, 1932. 5 vols.

DOCUMENTOS HISTORICOS 1659-1662. - Biblioteca Nacional. Vol. XX. Rio de Janeiro, 1930. 1 vol.

ECOLE LIBRE DES SCIENCES POLITIQUES (L'). Fondée en 1871 par Emile Boutmy. Paris, 1932. 1 vol.

EM MARCHA. Publicada por el Centro de Renovacion Cuenca-Ecuador. 1 vol.

ESCOLA REGIONAL (A). - Conferencia proferida durante a IVa. semana Nacional de Educação de 13 a 18 de Maio de 1931. 1 vol. 
ESTATISTICA INTELECTUAL DO BRASIL. - Rio de Janeiro, 1931. 2 vols.

ESTATISTICA ESCOLAR DE 1930. - Diretoria Geral do Ensino do Estado de S. Paulo. 1931. 2 vols.

EXPRESSÃO DE UM EXEMPLO (A). - S. Paulo, 1930. 1 vol.

FANTUZZI ROBERTO. - The form and conception in Painting of Robert Fantuzzi. Roma, 1930. 1 vol.

FUNDO DA QUESTÃO DA MANDCHURIA. - Broch. 1 vol.

JURISPRUDENCIA ELECTORAL. - Corte Electoral. Montevidéo, 1931. 1 vol.

LA POLITICA EXTERIOR DE LA REPUBLICA ARGENTINA. - Broch. 1 vol.

MANIFESTO DE LA UNIVERSIDAD MAYOR DE SAN FRANCISCO XAVIER DE CHUQUISACA CENTRAL DE BOLIVIA. - 1931. 1 vol.

MEMORIA DEL RECTOR DR. FRANCISCO GOMEZ DE LA TORRE. - Correspondiente al ano de 1931. Arequipa. Perú, 1932. 1 vol.

NOVOS ESTATUTOS. - Instituto Historico e Geografico de S. Paulo, 1932. 1 vol.

PROFISSIONAIS MATRICULADOS ATE' 31 DE DEZEMBRO DE 1926. - e durante os anos de 1927 a 1930. S. Paulo, 1931. 2 vols.

PROTESTO DEL GOBIERNO DE GUATEMALA ANTE EL INSTITUTO PAN AMERICANO DE GEOGRAFIA E HISTORIA. - Publicaciones de la Comission de Limites. Guatemala, 1932. 1 vol. 
RECENSEAMENTO DO BRASIL - realisado em $10^{\circ}$ de Dezembro de 1920. vol II (1a. parte). Rio de Janeiro, 1923. 1 vol.

REGULAMENTO DA ORDEM DOS ADVOGADOS BRASILEIROS. 1932.1 vol.

RELATORIO DO MINISTERIO DA FAZENDA. - Anos de 1907-1908. Rio de Janeiro. 4 vols.

RELATORIO DO MINISTERIO DA MARINHA. - Rio de Janeiro, 1907. 1 vol.

RELATORIO REFERENTE AOS SERVIÇOS FERROVIARIO E RODOVIARIO DA ESTRADA DE FERRO SOROCABANA. - relativo ao ano de 1931. S. Paulo, 1932. 1 vol.

RODOVIA 13 DE MAIO. - na serra do Martins. Rio de Janeiro, 1931. 1 vol.

SITUAÇÃO ECONOMICA DO ESTADO NO EXERCICIO DE 1926. - Secretaria das Finanças do Estado de Minas Gerais, Belo Horizonte, 1927. 1 vol.

SPEZIAL KATALOG. - Berne, 1924. 1 vol.

TECNICA REVOLUCIONARIA DO BOLCHEVISMO. Rio de Janeiro, 1927. 1 vol.

TERREMOTO DE NICARAGUA. - Informe oficial de la acción de socorros en Nicaragua despues del terremoto de 31 de Março de 1931. Washington, 1 vol.

UNIVERSIDADE DO PORTO. - Guia do Estudante. Porto. 1932. 1 vol. 